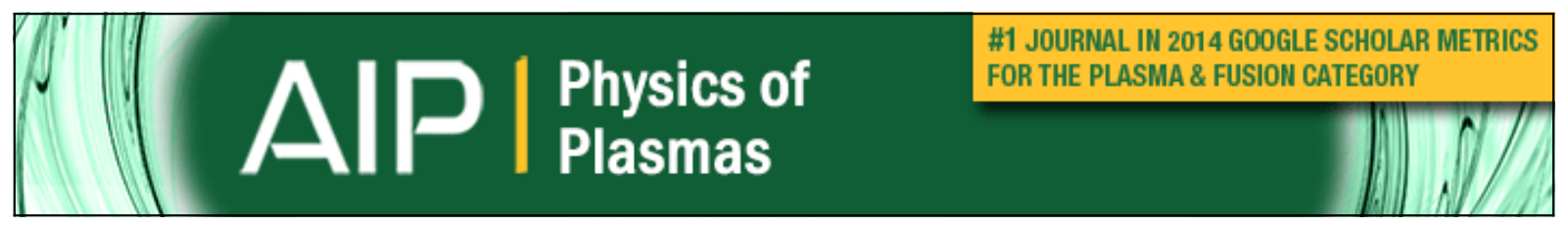

\title{
Grounded radio-frequency electrodes in contact with high density plasmas
}

A. Aanesland, C. Charles, R. W. Boswell, and M. A. Lieberman

Citation: Physics of Plasmas 12, 103505 (2005); doi: 10.1063/1.2089227

View online: http://dx.doi.org/10.1063/1.2089227

View Table of Contents: http://scitation.aip.org/content/aip/journal/pop/12/10?ver=pdfcov

Published by the AIP Publishing

\section{Articles you may be interested in}

Criteria of radio-frequency ring-shaped hollow cathode discharge using $\mathrm{H} 2$ and Ar gases for plasma processing J. Appl. Phys. 113, 033302 (2013); 10.1063/1.4776220

The discharge condition to enhance electron density of capacitively coupled plasma with multi-holed electrode Phys. Plasmas 19, 093508 (2012); 10.1063/1.4748576

Compression and strong rarefaction in high power impulse magnetron sputtering discharges

J. Appl. Phys. 108, 123306 (2010); 10.1063/1.3525986

The effects of radio-frequency bias on electron density in an inductively coupled plasma reactor J. Appl. Phys. 102, 113302 (2007); 10.1063/1.2815674

Kinetics driving high-density chlorine plasmas

J. Appl. Phys. 98, 063301 (2005); 10.1063/1.2037873

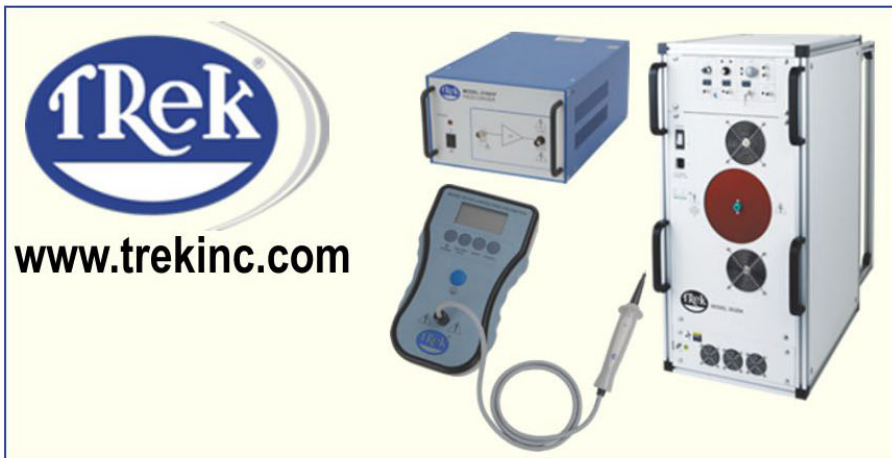

HIGH-VOLTAGE AMPLIFIERS AND ELECTROSTATIC VOLTMETERS

ENABLING RESEARCH AND INNOVATION IN DIELECTRICS, MICROFLUIDICS, MATERIALS, PLASMAS AND PIEZOS 


\title{
Grounded radio-frequency electrodes in contact with high density plasmas
}

\author{
A. Aanesland, ${ }^{\text {a) }}$ C. Charles, and R. W. Boswell \\ Plasma Research Laboratory, Research School of Physical Sciences and Engineering, \\ The Australian National University, ACT 0200, Australia \\ M. A. Lieberman \\ Department of Electrical Engineering and Computer Sciences, University of California, \\ Berkeley, California 94720
}

(Received 27 May 2005; accepted 6 September 2005; published online 17 October 2005)

\begin{abstract}
An analytical model is developed of an asymmetric electrode system immersed in a plasma, consisting of two dc-grounded electrodes, where the smaller one is biased at $13.56 \mathrm{MHz}$. The model is compared with a set of experiments performed in a high density low pressure plasma source (an electron cyclotron resonance source) where a second electrode is immersed into the plasma and powered by radio frequency. Excellent agreement is obtained between the analytical model and the experimental results. It is found that the time average plasma potential and the direct current (dc) flowing in the system during steady state are strongly dependent on both the rf voltage (or power) and the area ratio between the larger and smaller electrodes. For area ratios larger than 80, the dc current is large and the plasma potential is constant with respect to the applied rf voltage. For area ratios smaller than 80 but larger than unity, the plasma potential increases linearly with the applied rf voltage, and the dc current is reduced compared to the large area ratio case. (C) 2005 American Institute of Physics. [DOI: 10.1063/1.2089227]
\end{abstract}

\section{INTRODUCTION}

In most fields of plasma physics the understanding of the plasma potential formation is crucial, as it plays a significant role in many nonlinear phenomena such as particle confinement, particle acceleration, and transport barriers. Here can be mentioned a wide range of fields from laboratory to space, e.g., the acceleration of the primary aurora electrons, ${ }^{1}$ the acceleration of ions in plasma thruster technology, ${ }^{2}$ or the additional ion or plasma heating in fusion devices, ${ }^{3,4}$ which all utilize the formation and/or control of the plasma potential. Also the knowledge of the plasma sheaths in general and in radio frequency (rf) fields in particular is important for the success of controlled plasma processing, such as reactive ion etching. ${ }^{5}$

When a rf signal is applied to a surface in contact with the plasma the sheath formed between this surface and the plasma will oscillate at a frequency corresponding to the applied frequency. If the applied voltage amplitude is large, the sheath oscillations might influence the bulk plasma such that the global plasma potential oscillates as well. ${ }^{6}$ Hence, not only the sheath in front of the electrode oscillates, but the bulk plasma and all other sheaths in front of all surfaces in contact with the plasma might be influenced by the applied signal. The sheath dynamics has been investigated in depth by many authors, and a detailed review of the ion energy distribution and the ion behavior in the rf sheath is given by Kawamura et al. ${ }^{7}$

In plasma processing the substrate (or electrode) is usually dc isolated from ground via a blocking capacitor, which charges up negatively in asymmetric discharges, and a selfbias is formed on the substrate. This phenomenon is essen-

\footnotetext{
${ }^{a)}$ Electronic mail: ane.aanesland@anu.edu.au
}

tially the same as that described by Butler and Kino, ${ }^{8}$ and has been thoroughly investigated both experimentally and by computer simulations. ${ }^{9-11}$

In most magnetically confined fusion plasmas, additional antennae are required to increase the plasma temperature. Launching waves in the ion cyclotron frequency range is one of the rf heating techniques that are applied successfully. ${ }^{3,12,13}$ As an example, the rf antenna in the H-1 Heliac experiment at the Australian National University, is shaped like a loop in the plane of the last closed flux surface (LCFS) and is in contact with the plasma. ${ }^{4}$ In this case one end of the antenna loop is grounded, which allows a direct current $(\mathrm{dc})$ to flow from the plasma via the antenna to ground. Currents of around 90 amperes and a plasma potential of 100 volts at the LCFS have been measured for typical experimental conditions in this device. Also, in smaller systems where the immersed live electrode is dc grounded, large plasma potentials have been measured, and simultaneously arcing and fluctuations in the plasma were observed. ${ }^{14-17}$ This phenomenon is not yet fully understood.

Here we describe an analytical model of a plasma with an asymmetric electrode system, where an immersed small live electrode is dc grounded and allows a direct current to flow from the plasma via the electrodes to ground during steady state. The effect of the rf voltage applied to the live electrode and of the area ratio between the grounded electrode/wall and the live electrode is investigated, with particular emphasis on the time average plasma potential and on the dc current flowing in the system during steady state. The results from this analytical model are compared with a set of experiments performed in an electron cyclotron resonance (ECR) source which has been modified by inserting a live rf biased electrode into and in direct contact with the 


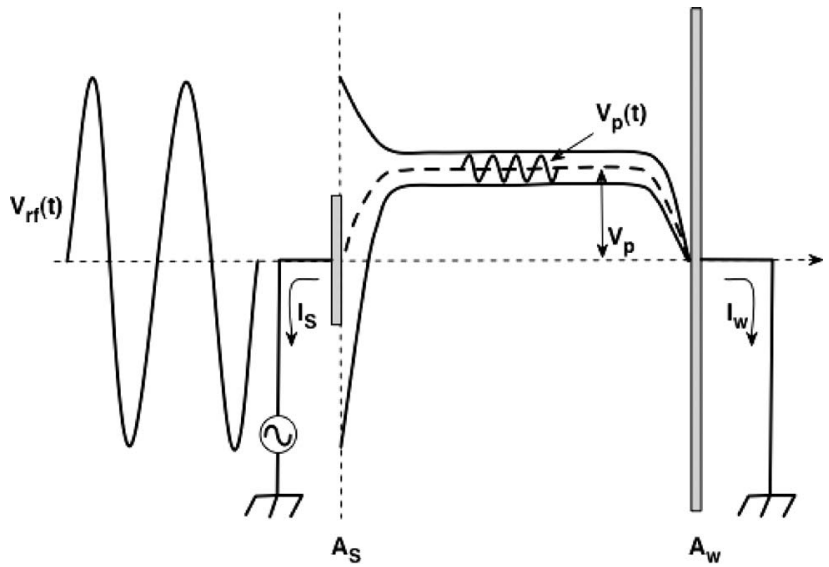

FIG. 1. Illustration of the voltage distribution in front of the live electrode (area $A_{s}$ ) and the wall $\left(\operatorname{area} A_{w}\right.$ ) at various times within the rf cycle. Both electrodes are grounded and in direct contact with the plasma.

ECR-generated plasma. The average plasma potential and the dc current to the live electrode is measured as a function of the applied rf voltage for different area ratios between the live and grounded electrode.

\section{MODEL OF IMMERSED ELECTRODE}

\section{A. Model schematic/description}

We consider an asymmetric electrode system immersed in a plasma illustrated on Fig. 1, which consists of two electrodes. The left electrode of area $A_{s}$ is powered by an $\mathrm{rf}$ voltage of amplitude $V_{r f}$, while the right electrode of area $A_{w}$ is grounded. Both electrodes are metallic and in direct contact with the plasma. The live electrode is also dc grounded. The electrical circuit is therefore closed and a dc current is allowed to flow from the plasma via the electrodes to ground during steady state. In this model we investigate how the direct current, $I_{d c}=\left|I_{w}\right|=\left|I_{S}\right|$, and the averaged plasma potential, $V_{p}$, are affected by the applied rf voltage on the small live electrode for different area ratios, $A_{w} / A_{s}$, between the grounded electrode/wall and the smaller live electrode. The live electrode is considered to be small with relatively low rf power applied to it: the plasma is generated by an external $\mathrm{rf}$ or microwave source, which is not considered in this model.

\section{B. Voltage distribution in the system}

The voltage distribution in the system is illustrated in Fig. 1, for various times in the rf cycle. Assuming that the electrons are sufficiently mobile, all the voltage drop occurs mainly across the sheaths in front of the electrodes, and the electric fields in the bulk plasma can be neglected. As illustrated in Fig. 1, when the applied rf voltage, $V_{r f}(t)$ $=V_{r f} \cos (\omega t)$, is at its maximum, the instantaneous plasma potential, $V_{p}(t)$, is also at its maximum and can be lower than the rf amplitude, $V_{r f}$, at the live (smaller) electrode, resulting in an electron current flow from the plasma to the electrode. When $V_{r f}(t)$ is most negative, $V_{p}(t)$ is much larger than $V_{r f}$, and an ion current flows from the plasma to the electrode. For a large area ratio, $A_{w} / A_{s}$, the rf voltage drop appears mainly across the sheath in front of the live electrode $\left(A_{s}\right)$,

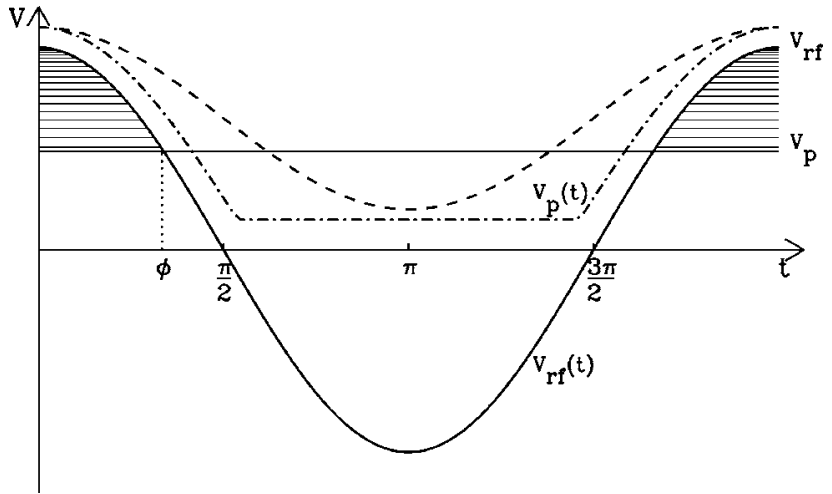

FIG. 2. Illustration of the applied rf voltage $V_{r f}(t)$ (solid line) on the live electrode, and the response of the oscillating plasma potential $V_{p}(t)$, depending on the sheath impedance. The dashed line represents a capacitive sheath and the dashed-dotted line represents a resistive sheath (Ref. 19). The hatched area shows the fraction of time, $\phi$, when the electrons can escape to the grounded electrode.

and the plasma potential (with respect to the grounded wall) has a small rf component compared to its dc component. The waveform of the live electrode-to-plasma voltage is strongly affected by the sheath dynamics. ${ }^{18,19}$ Assuming that the average voltage drop across the sheath is much larger than the typical voltage drop in the presheath, $\sim 0.5 k T_{e}$, the initial ion velocity entering the sheath, i.e., the ion sound speed, can be neglected. The ion transit time through the sheath is then given by ${ }^{7}$

$$
\tau_{i o n}=3 \bar{s}\left(\frac{m_{i}}{2 e \bar{V}_{s}}\right)^{1 / 2},
$$

where $\bar{s}$ is the time average sheath thickness, $m_{i}$ is the ion mass, $e$ is the electron charge and $\bar{V}_{s}$ is the mean sheath voltage. Inserting the typical plasma parameters obtained in the experiments discussed in Sec. III, an ion transit time of about $50-100 \mathrm{~ns}$ is found, which is of the order of the $\mathrm{rf}$ period at $13.56 \mathrm{MHz}, \tau_{r f}=74 \mathrm{~ns}$. At high frequencies, $\tau_{i o n} / \tau_{r f} \gg 1$ and the displacement current is larger than the conduction current through the sheath: the sheath behaves capacitively. At low frequencies, $\tau_{\text {ion }} / \tau_{r f} \ll 1$ and the conduction current dominates so that the sheath can be described as resistive in nature. In our analytical model, where $\tau_{i o n} / \tau_{r f}$ $\approx 1$, we assume that the sheath in front of the live electrode is a combination of a resistive and capacitive sheath. ${ }^{19}$ The applied rf voltage and the corresponding plasma potential oscillations have roughly the waveforms shown in Fig. 2, where the dashed and dash-dotted lines represent a capacitive $\left(\tau_{i o n} / \tau_{r f} \gg 1\right)$ and resistive $\quad\left(\tau_{i o n} / \tau_{r f} \ll 1\right)$ sheath, respectively. 16,18

Figure 2 shows that the instantaneous plasma potential, $V_{p}(t)$, approaches the instantaneous rf voltage, $V_{r f}(t)$, during the positive excursion of the rf cycle, in particular when $V_{r f}(t)$ is larger than the time average plasma potential $V_{p}$. We assume that the electrons reach the grounded electrode only during this time, i.e., when 


$$
V_{r f} \cos (\omega t)-V_{p}>0 .
$$

As indicated by the hatched areas in Fig. 2, the electrons reach the electrode only during a fraction of the $\mathrm{rf}$ period $(\phi / \pi)$, where $\phi$ can be expressed as

$$
\phi=\cos ^{-1}\left(\frac{V_{p}}{V_{r f}}\right) \text {. }
$$

When the average plasma potential approaches the rf amplitude, the fraction of time when the electrons can reach the electrode becomes very small and approaches zero $\left(V_{p} / V_{r f}\right.$ $\rightarrow 1, \phi \rightarrow 0)$. On the other hand, when $V_{p}$ is much less than $V_{r f}$ the electrons can be collected at the positive part of the $\mathrm{rf}$ cycle, corresponding to half the rf period $\left(V_{p} / V_{r f} \rightarrow 0, \phi\right.$ $\rightarrow \pi / 2)$.

\section{Dc current through the system}

As shown above, the electron current to the live electrode, $I_{e s}$, during one $\mathrm{rf}$ period is the flux of electrons reaching the electrode during a fraction, $\phi / \pi$, of the rf period, which can be expressed as

$$
I_{e s}=\frac{1}{4} e n_{0} \bar{v}_{e} A_{s} \frac{\phi}{\pi}
$$

where $n_{0}$ is the bulk plasma density and $\bar{v}_{e}=\sqrt{8 k T_{e} / \pi m_{e}}$ is the mean electron speed. The ions are flowing to the live electrode during the entire rf cycle and contribute to an ion current, $I_{i s}$ given by ${ }^{20}$

$$
I_{i s}=0.61 e n_{0} C_{s} A_{s},
$$

where the ions enter the electrode sheath with the ion sound speed $C_{s}=\sqrt{k T_{e} / m_{i}}$. The total current to the live electrode, $I_{s}$ is a sum of the electron and ion currents

$$
I_{s}=0.61 e n_{0} C_{s} A_{s}-\frac{1}{4 \pi} e n_{0} \bar{v}_{e} A_{s} \cos ^{-1}\left(\frac{V_{p}}{V_{r f}}\right) .
$$

At the large grounded electrode, we assume that the rf component of the plasma potential (with respect to ground) is small compared to the dc component. In this case the current to the grounded electrode, $I_{w}$, can be expressed as a steady flow of ions, $I_{i w}$, and electrons, $I_{e w}$, given by

$$
I_{w}=0.61 e n_{0} C_{s} A_{w}-\frac{1}{4} e n_{0} \bar{v}_{e} A_{w} \exp \left(-\frac{e V_{p}}{k T_{e}}\right) \text {. }
$$

\section{The effect on the plasma potential}

As the dc circuit is closed the current to the live and grounded electrode must be equal, $I_{s}=I_{w}$. A relation between the voltage ratio, $V_{p} / V_{r f}$, and the area ratio, $A_{w} / A_{s}$, is found by equating Eqs. (6) and (7). Neglecting the ion current in Eq. (6) compared to the ion current in Eq. (7), we obtain

$$
\cos ^{-1}\left(\frac{V_{p}}{V_{r f}}\right)=\pi\left[0.61\left(\frac{2 \pi m}{M}\right)^{1 / 2}-\exp \left(-\frac{e V_{p}}{k T_{e}}\right)\right] \frac{A_{w}}{A_{s}}
$$

Figure 3 shows $V_{p} / V_{r f}$ as a function of the area ratio $A_{w} / A_{s}$, using Eq. (8). Five values of $V_{r f}$ are used $(20,60$, 100,200 , and $300 \mathrm{~V}$ ), where the solid line represents $20 \mathrm{~V}$

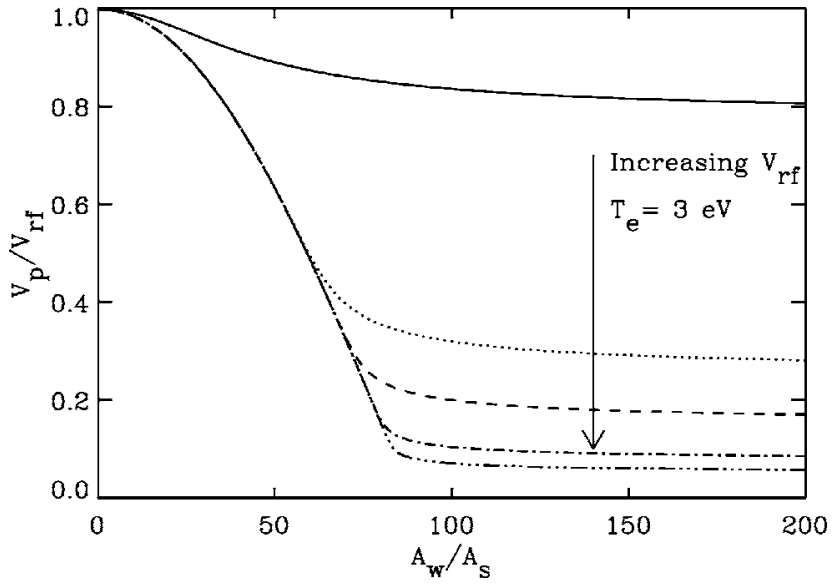

FIG. 3. The plasma potential $V_{p}$ normalized to the rf voltage $V_{r f}$ as a function of the area ratio between the grounded electrode/wall area $\left(A_{w}\right)$ and the live electrode area $\left(A_{s}\right)$. The rf amplitude is changed from $20 \mathrm{~V}$ (solid line) to $60,100,200$, and $300 \mathrm{~V}$ (dashed-triple-dotted line), while the electron temperature is constant at $3 \mathrm{eV}$.

and the dashed-triple-dotted line represents $300 \mathrm{~V}$. The electron temperature is kept constant at $3 \mathrm{eV}$. The two zones shown in the figure (i) $A_{w} / A_{s} \geqslant 80$ and (ii) $1<A_{w} / A_{s} \leqslant 80$ are discussed below.

(i) $A_{w} / A_{s} \geqslant 80$ : the live electrode area is at least two orders of magnitude smaller than that of the grounded electrode. In this case $V_{p}$ approaches an asymptote $\left(V_{p 0}\right)$ for all the applied rf voltages, implying that when the live electrode becomes very small, its effect on the plasma potential is limited. The asymptotic value can be found by setting the right side of Eq. (8) equal to zero, which gives

$$
V_{p 0}=-k T_{e} \ln \left[0.61\left(\frac{2 \pi m}{M}\right)^{1 / 2}\right] \text {. }
$$

For a $3 \mathrm{eV}$ argon plasma, $V_{p 0}=15.5 \mathrm{~V}$, which corresponds to a standard sheath voltage in front of a grounded wall where almost equal electron and ion currents flow to the wall, i.e., $I_{w}=0$. In this case $\phi \rightarrow \pi / 2$ and the direct current is limited to the flow of electrons collected by the live electrode over half of an rf cycle,

$$
I_{d c} \approx-\frac{1}{8} e n_{0} \bar{v}_{e} A_{s} .
$$

(ii) $1<A_{w} / A_{s} \leqslant 80$ : the live electrode area is smaller than the grounded electrode area, but not two orders of magnitude smaller. Then the voltage ratio $V_{p} / V_{r f}$ increases when the area ratio $A_{w} / A_{s}$ decreases. Hence, the live electrode affects the plasma potential dramatically, and $V_{p}$ can increase to the order of the applied rf voltage. In this case $\phi$ adjusts itself such that the dc current is limited to the flow of ions collected by the grounded electrode over an entire rf cycle,

$$
I_{d c}=-0.61 e n_{0} C_{s} A_{w} .
$$

Let us now keep the area ratio, $A_{w} / A_{s}$, constant to investigate the relation between $V_{p}$ and $V_{r f}$. Both $V_{p}$ and $V_{r f}$ are unknown values in this system, so for simplicity Eq. (8) is solved with respect to $V_{r f}$, although $V_{r f}$, and not $V_{p}$, is the experimental control parameter. The result is shown in Fig. 4 , where the average plasma potential, $V_{p}$ is plotted as a 


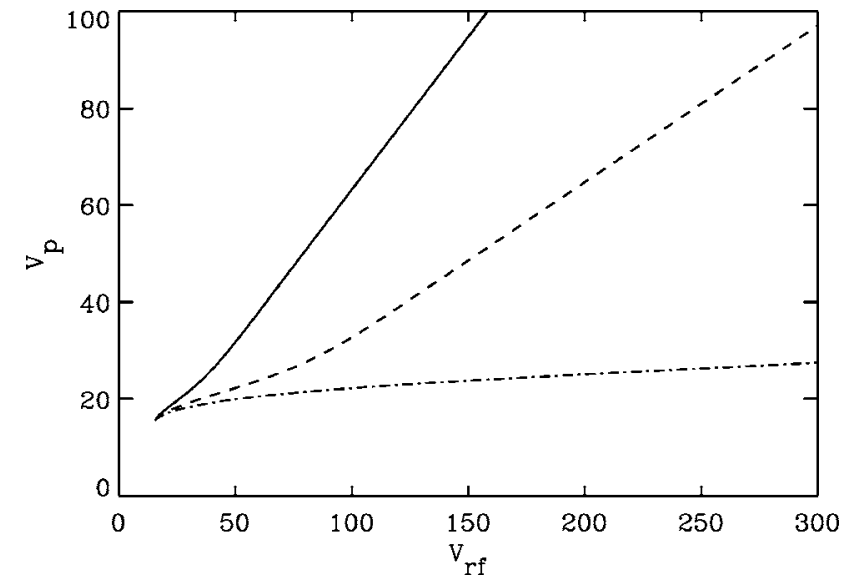

FIG. 4. The plasma potential $V_{p}$ as a function of the applied rf voltage $V_{r f}$ on the live electrode, calculated using Eq. (8) with area ratios of $A_{w} / A_{s}$ $=50$ (solid line), $A_{w} / A_{s}=70$ (dashed line), and $A_{w} / A_{s}=85$ (dash-dotted line).

function of the applied rf voltage, $V_{r f}$. The solid, dashed, and dash-dotted lines represent fixed area ratios of 50, 70, and 85 , respectively. For a small area ratio, there is a strong linear dependency between the applied rf voltage and the plasma potential, while for a large area ratio, $V_{p}$ is almost constant with the applied voltage, in agreement with the preceding discussion.

\section{E. Dc current to the live electrode}

The dc current flowing to the live electrode depends both on $A_{s}$ and on the area ratio $A_{w} / A_{s}$, and can be found using Eq. (6) by utilizing the relation between $V_{r f}$ and $V_{p}$ found in the previous section (Fig. 4). Figure 5 shows the direct current, $I_{d c}=I_{s}$, as a function of the rf voltage on the live electrode for an area $A_{s}=40 \mathrm{~cm}^{2}$. As in Fig. 4, the solid, dashed and dash-dotted lines represent fixed area ratios of 50, 70, and 85 , respectively. In this calculation an electron temperature of $3 \mathrm{eV}$ and a plasma density of $0.5 \times 10^{11} \mathrm{~cm}^{-3}$ are used, which are the average values measured by the Langmuir probe described in Sec. III A. Note that the calculation starts for $V_{r f}=V_{p 0}$ as there is no solution below this value.

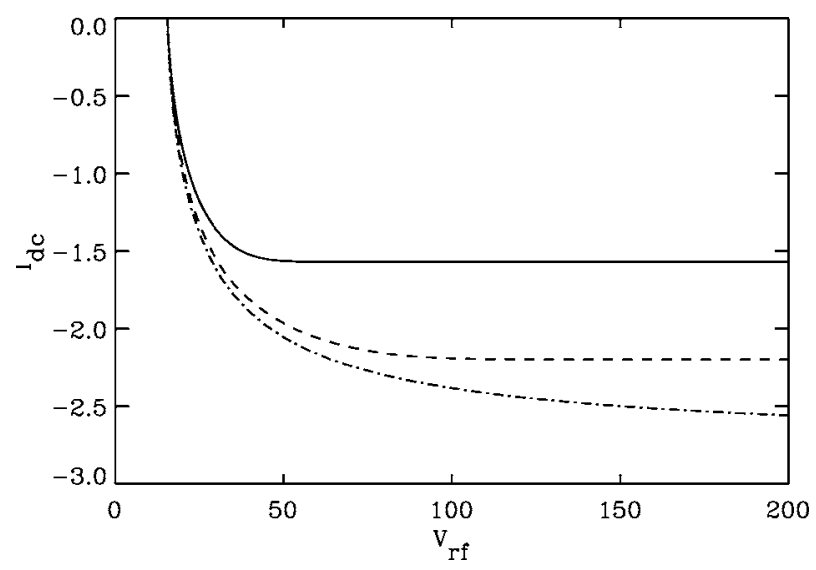

FIG. 5. The direct current $I_{d c}$ to the live electrode as a function of the applied rf voltage for $A_{s}=40 \mathrm{~cm}^{2}$ and area ratios of $A_{w} / A_{s}=50$ (solid line), $A_{w} / A_{s}=70$ (dashed line), and $A_{w} / A_{s}=85$ (dash-dotted line). The electron temperature is $3 \mathrm{eV}$ and the plasma density is $0.5 \times 10^{11} \mathrm{~cm}^{-3}$.
Figure 5 shows that the dc current flowing to the live electrode is negative, hence, electrons are flowing to the live electrode while ions are flowing to the grounded electrode during steady state. This current increases with the applied rf voltage on the live electrode. The current is larger for an area ratio of 85 than for the area ratio of 50 corresponding to an increase in $A_{w}$ at a fixed $A_{s}$. The current reaches a limit of $1.5 \mathrm{~A}$ in the case of $A_{w} / A_{s}=50$, while it is $2.7 \mathrm{~A}$ in the case of $A_{w} / A_{s}=85$.

\section{F. Preliminary discussion}

For large area ratios (85), the plasma potential is rather constant with respect to the applied rf voltage, and the dc current is large. For small area ratios (50), the plasma potential increases linearly with the applied rf voltage, and the dc current (at a fixed $A_{s}$ ) is reduced. This can be understood from Fig. 1: when $V_{p}$ is constant and $V_{r f}$ increases, $\phi / \pi$ increases, and consequently the electrons are able to reach the live electrode during a larger fraction of the rf period. On the contrary, when $V_{p}$ increases with $V_{r f}$, the fraction $\phi / \pi$ remains constant or can even decrease with $V_{r f}$, and the dc current is therefore constant with increasing $V_{r f}$. In the latter case, the effect of the live electrode is only seen by the increase in the plasma potential, while in the first case it is mainly seen by the large dc current flowing in the system.

\section{EXPERIMENTAL RESULTS}

\section{A. Experimental setup}

The electron cyclotron resonance (ECR) source, "Menja," shown in Fig. 6, has been described previously. ${ }^{21,22}$ Briefly, $\mathrm{TE}_{11}$ microwaves at $2.45 \mathrm{GHz}$ are fed into the vacuum chamber via a circular quartz window. A divergent magnetic field is applied, placing the ECR resonance, at $875 \mathrm{G}, 12 \mathrm{~cm}$ from the window in the narrow part of the chamber (source). In the present study, the experiment is modified by inserting a one loop copper rf electrode inside the vacuum chamber at the exit of the source, $22 \mathrm{~cm}$ from the microwave window and $10 \mathrm{~cm}$ from the ECR zone. The diameter of the antenna loop is $7 \mathrm{~cm}$ and the copper wire is $0.6 \mathrm{~cm}$ diameter giving a surface area of $A_{s}=4 \pi^{2} \cdot 0.3 \cdot 3.5$ $\approx 40 \mathrm{~cm}^{2}$. Rf power at $13.56 \mathrm{MHz}$ is fed to the electrode via a matching network, and the forward and reflected power is monitored by a Bird watt-meter. The copper electrode is directly in contact with the plasma and dc grounded by an inductor placed inside the match box, allowing a direct current to flow from the plasma via the electrode to ground, as discussed in the previous section. This current, $I_{d c}$, is measured using an amp-meter connected to the rf antenna inside the match box. A Langmuir probe (LP) is used to acquire the plasma potential $\left(V_{p}\right)$, and the acquisition system and analysis have been described in detail elsewhere. ${ }^{21,22}$ The LP is inserted on axis of the chamber and is situated in the center of the immersed rf electrode. As LP characteristics might be altered by a rf oscillating sheath around the probe, an emissive probe was used to confirm the validity of the plasma potential acquired by the LP; an agreement within $10 \%$ was obtained. 


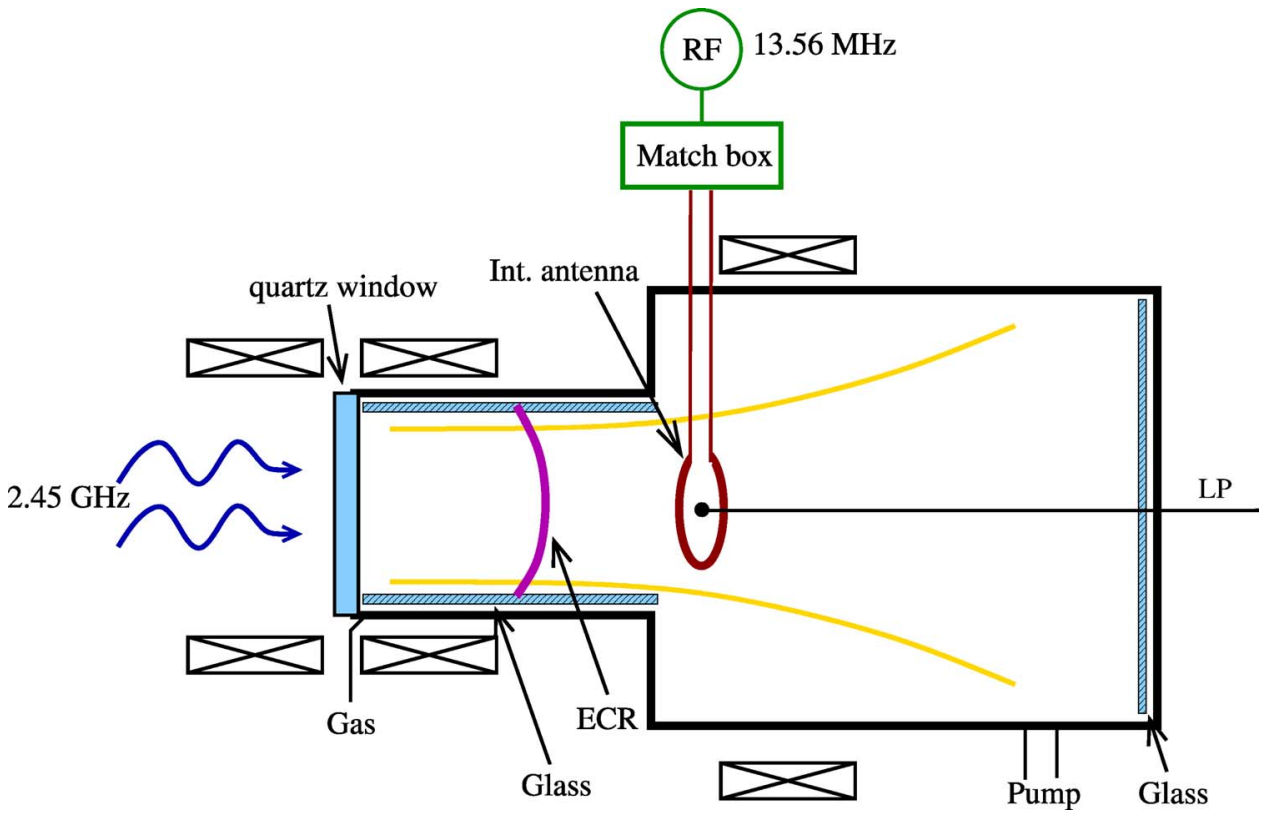

FIG. 6. A schematic drawing of the ECR source, "Menja." The $\mathrm{TE}_{11}$ mode enters from the left, and reaches the ECR resonance in the source. The rf electrode is inserted in the diffusion chamber $10 \mathrm{~cm}$ from the ECR zone. Glass is inserted in the source and the back plate of the diffusion chamber in some of the experiments, as specified in the text.
As discussed in the previous section, the effect of a live electrode immersed in the plasma strongly depends on the area ratio between the electrode, with area $A_{s}$, and the area of the grounded wall in the system, $A_{w}$. To test our model, two experimental configurations are used. First, the live electrode is immersed in a completely grounded vacuum vessel, which is the normal configuration in ECR sources, giving an area ratio $A_{w} / A_{s}=85$ between the grounded wall and the live rf electrode. Second, the source and the end wall of the chamber are isolated from ground by a $3 \mathrm{~mm}$ glass cylinder and plate, as illustrated in Fig. 6, giving an area ratio $A_{w} / A_{s}$ $=70$.

The argon pressure is $0.5 \mathrm{~Pa}(3.8 \mathrm{mTorr})$, the magnetic field at the LP position, and electrode position is $600 \mathrm{G}$, the microwave power is $500 \mathrm{~W}$, and the rf power on the live electrode is varied from 0 to $100 \mathrm{~W}$. The plasma is therefore mainly generated by the microwave source, the main assumption in our analytical model.

\section{B. Results}

The plasma potential, $V_{p}$, and the direct current to the live electrode, $I_{d c}$, are measured as a function of the applied rf power on the immersed electrode, and the results are shown in Figs. 7 and 8, respectively. The open diamonds are obtained when the whole vacuum chamber is grounded, and represent an area ratio $A_{w} / A_{s}=85$, while the closed diamonds are obtained when the source is floating and represents an area ratio of $A_{w} / A_{s}=70$.

With an area ratio of 85 (open diamonds), $V_{p}$ is fairly constant at $15 \mathrm{~V}$ and $I_{d c}$ decreases from 0 to $-2.5 \mathrm{~A}$. With the area ratio of 70 (closed diamonds), $V_{p}$ increases "linearly" from 10 to $30 \mathrm{~V}$, and $I_{d c}$ decreases from 0 to $-1.5 \mathrm{~A}$.

\section{Comparison between analytical model and experiment}

To fully compare our analytical model and the experimental results performed in the ECR source a relation be- tween the input rf voltage (model) and the rf power (experiment) is required. One possibility is to calculate the relation analytically as discussed in detail in Ref. 20, but this requires strong assumptions on the power absorption mechanism into the system. The relation between the rf power and the rf voltage can also be found by a new set of experiments. In this experiment we isolate the live electrode from ground by a $10 \mathrm{nF}$ blocking capacitor. As described in the Introduction, a negative self-bias will now form, preventing direct current flow during steady state. The self-bias, $V_{d c}$, is related to the applied rf voltage via the sheath capacitances at the live and grounded electrode. ${ }^{16}$ As the capacitance of the sheath is determined by the area and the sheath thickness, $(C$ $\left.=\epsilon_{0} A / d\right)$, an expression of the self-bias can be found:

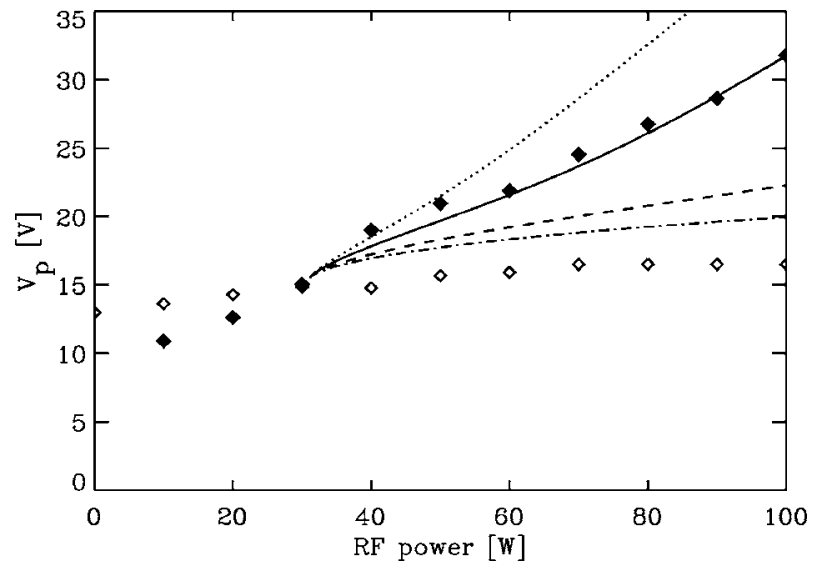

FIG. 7. Plasma potential as a function of rf power on the immersed electrode. Open diamonds are obtained with the source wall grounded $\left(A_{w} / A_{s}\right.$ $=85$ ), while closed diamonds are obtained with a dielectric source wall $\left(A_{w} / A_{s}=70\right)$. Dotted, solid, dashed, and dashed-dotted lines represent the analytical model from Fig. 4 and Eq. (8), with area ratios 35, 50, 70, and 85, respectively. 


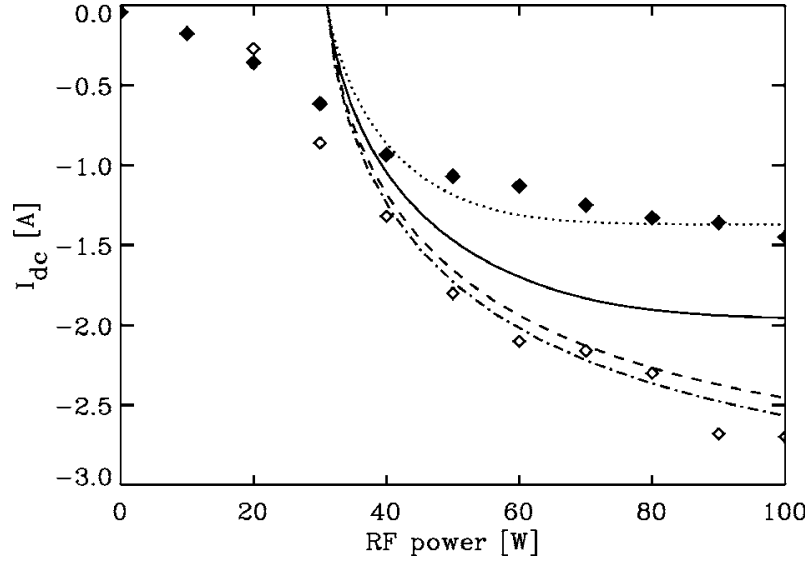

FIG. 8. Current to the grounded electrode as a function of rf power on the immersed electrode. Open diamonds are obtained with the source wall grounded $\left(A_{w} / A_{s}=85\right)$, while closed diamonds are obtained with a dielectric source wall $\left(A_{w} / A_{s}=70\right)$. Dotted, solid, dashed, and dashed-dotted lines represent the analytical model from Fig. 5 and Eq. (6), with area ratios 35, 50, 70 , and 85 , respectively.

$$
V_{d c}=V_{r f}\left(\frac{d_{w} / d_{s}-A_{w} / A_{s}}{d_{w} / d_{s}+A_{w} / A_{s}}\right)
$$

where $d_{w}$ and $d_{s}$ are the sheath thickness in front of grounded wall and live electrode, respectively. $d_{w}$ and $d_{s}$ can be found using Child-Langmuir's law where $d \propto V^{3 / 4}$. Figure 9 shows the self-bias measured as a function of the rf power applied to the live electrode, and since the self-bias in this system is relatively low, $d_{w} / d_{s} \ll A_{w} / A_{s}$, and we find from Eq. (12) that $V_{d c} \approx-0.98 V_{r f}$. A linear dependency was found between $V_{d c}$ and $P_{r f}$ for both $A_{w} / A_{s}=70$ and $A_{w} / A_{s}=85$, where the selfbias is $-50 \mathrm{~V}$ at $100 \mathrm{~W}$ giving a gradient of 0.5 . The applied rf voltage in the model and the applied rf power in the experiments are therefore related by

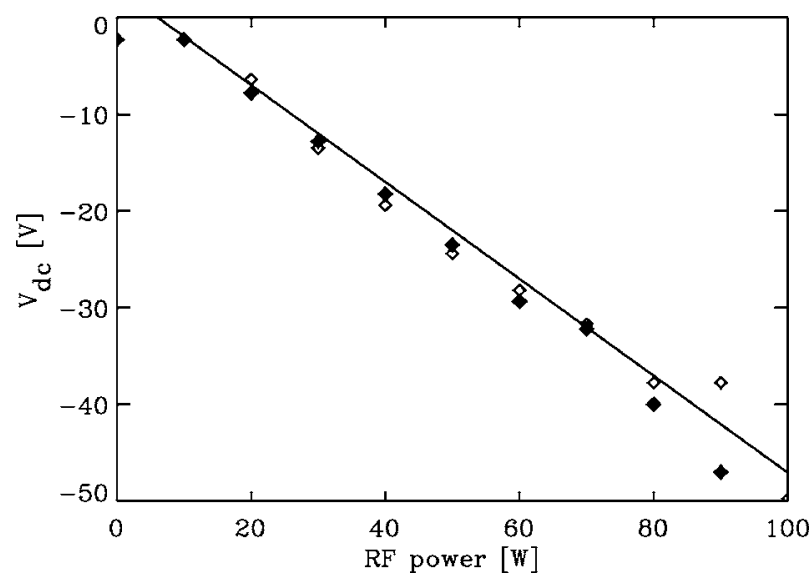

FIG. 9. The self-bias as a function of rf power on the immersed electrode, when a $10 \mathrm{nF}$ capacitor is placed between the live electrode and ground. Open diamonds are obtained with the source wall grounded $\left(A_{w} / A_{s}=85\right)$, while closed diamonds are obtained with a dielectric source wall $\left(A_{w} / A_{s}\right.$ $=70$ ).

$$
\frac{V_{r f}}{V_{0}}=\frac{1}{2} \frac{P_{r f}}{P_{0}},
$$

where $V_{0}$ and $P_{0}$ correspond to $1 \mathrm{~V}$ and $1 \mathrm{~W}$, respectively. The solid, dashed and dashed-dotted line in Figs. 7 and 8 are the results from the analytical model [Eqs. (8) and (6)], corresponding to the results in Figs. 4 and 5, but now plotted as a function of the rf power.

An extremely good agreement between the analytical model and the experimental results in Menja, are obtained. The plasma potential increases with rf power for a small area ratio and is fairly constant for larger area ratios, and the dc current to the electrode decreases in a similar manner in both the analytical model and the experiment. The calculated plasma potential with an area ratio of $A_{w} / A_{s}=50$ (solid line) and the measured values for the smaller area ratio, $A_{w} / A_{s}$ $=70$, in the experiments (filled diamonds) are in very good agreement. To calculate the area ratio in Menja, $A_{w}$ was taken as the total surface area of the vacuum chamber consisting of metallic grounded walls. We have assumed that the ion fluxes impinging upon all grounded surfaces are equal so the walls in the different areas of the vacuum chamber are of equal importance. Hence, the influence of the magnetic field, making some grounded areas more important than others, is not taken into account. The actual area ratio in the experiment when glass is introduced is therefore presumably less than what we calculate, considering that the ion flux to the source wall and to the back, downstream side, of the chamber might be larger than the ion flux to the side walls in the diffusion chamber. The same argument is valid for the measured and calculated dc current, where the agreement between the results obtained with the insulated source wall is in very good agreement with the analytical model using an area ratio of 35-50. The measured and calculated current in the totally grounded vacuum chamber is in very good agreement with an area ratio around $70-85$.

\section{CONCLUSION}

An analytical model of a very asymmetric electrode system is developed which shows very good agreements with experimental results. In this study, both the small live electrode and the large grounded electrode are immersed into the plasma, and a direct current is allowed to flow during steady state. The effects of both the applied rf voltage/power and the area ratio between the electrodes on the final equilibrium plasma parameters have been discussed. It was found that for very large area ratios, where the live electrode is at least two orders of magnitude smaller than the large electrode, the average plasma potential is rather constant with increasing $\mathrm{rf}$ voltage. In this case the electrons are able to reach the live electrode during a large fraction of the rf period, and the direct current flowing in the system is limited by this flow of electrons. When the area ratio decreases, but is still asymmetric, the plasma potential increases linearly with the applied rf voltage. The electrons are now able to reach the live electrode during a constant fraction of the rf period, independent on the rf voltage. In this case it was found that the direct current is limited by the flow of ions to the grounded wall. 
In this first attempt at modeling these very asymmetric discharges, the simplest model was developed which assumes that the electron current to the live electrode is given by the rf voltage amplitude and the average plasma potential [Eq. (2)]. The very good agreement between the model and the experiment suggests the validity of this assumption on the first approximation. However, given the very high electron mobility, the electron current should depend on the instantaneous potential difference between the rf voltage, $V_{r f}(t)$, and the instantaneous plasma potential, $V_{p}(t)$, rather than $V_{r f}$ and the average $V_{p}$ as assumed in Eq. (2). Future work will include this instantaneous potential difference between $V_{r f}(t)$ and $V_{p}(t)$ in calculating $\phi$.

The results shown here are relevant to fusion experiments, where grounded-immersed antennas are used to launch waves in the ion cyclotron frequency range for ion heating. As we have shown here, the antenna can, depending on its size, generate an increase of the plasma potential along the field lines intercepting the antenna, and as a result strongly alter the particle confinement and increase the crossfield diffusion. It may also draw very large currents from the plasma, hence reducing the plasma density.

${ }^{1}$ R. E. Ergun, Y.-J. Su, L. Andersson, C. W. Carlson, J. P. McFadden, F. S. Mozer, D. L. Newman, M. V. Goldman, and R. J. Strangeway, Phys. Rev. Lett. 87, 045003 (2001).

${ }^{2}$ J. M. Haas and A. D. Gallimore, Phys. Plasmas 8, 652 (2000).

${ }^{3}$ D. A. D'Ippolito, J. R. Myra, J. Jacquinot, and M. Bures, Phys. Fluids B 5, 3603 (1993).
${ }^{4}$ M. G. Shats, D. L. Rudakov, R. W. Boswell, and G. G. Borg, Phys. Plasmas 4, 3629 (1997).

${ }^{5}$ A. J. Perry and R. W. Boswell, Appl. Phys. Lett. 55, 148 (1989).

${ }^{6}$ J. W. Coburn and E. Kay, J. Appl. Phys. 43, 4965 (1972).

${ }^{7}$ E. Kawamura, V. Vahedi, M. A. Lieberman, and C. K. Birdsall, Plasma Sources Sci. Technol. 8, 45 (1999).

${ }^{8}$ H. S. Butler and G. S. Kino, Phys. Fluids 6, 1346 (1963).

${ }^{9}$ T. Fukasawa, T. Nouda, A. Nakamura, H. Shindo, and Y. Horiike, Jpn. J. Appl. Phys. 32, 6076 (1993).

${ }^{10}$ J.-H. Kim, Y.-H. Shin, and K.-H. Chung, Thin Solid Films 435, 288 (2003).

${ }^{11}$ H. B. Smith, C. Charles, R. W. Boswell, and H. Kuwahara, J. Appl. Phys. 82, 561 (1997).

${ }^{12}$ J. H. Harris, T. Hutter, J. T. Hogan, V. Basiuk, B. Beaumont, A. Becoulet, S. Bremond, M. D. Carter, M. Goniche, R. H. Goulding, D. Guilhem, G. R. Haste, D. J. Hoffman, X. Litaudon, and F. Nguyen, J. Nucl. Mater. 241-243, 511 (1997).

${ }^{13}$ J. M. Noterdaeme and G. Van Oost, Plasma Phys. Controlled Fusion 35, 1481 (1993).

${ }^{14}$ A. Aanesland, C. Charles, and R. W. Boswell, IEEE Trans. Plasma Sci. 33, 360 (2005).

${ }^{15}$ A. Aanesland, C. Charles, R. W. Boswell, and Å. Fredriksen, J. Phys. D 37, 1334 (2004).

${ }^{16}$ K. Köhler, J. W. Coburn, D. E. Horne, E. Kay, and J. H. Keller, J. Appl. Phys. 57, 59 (1985)

${ }^{17}$ H. Sugai, K. Nakamura, and K. Suzuki, Jpn. J. Appl. Phys. 33, 2189 (1994).

${ }^{18}$ R. H. Bruce, J. Appl. Phys. 52, 7064 (1981).

${ }^{19}$ K. Köhler, D. E. Horne, and J. W. Coburn, J. Appl. Phys. 58, 3350 (1985).

${ }^{20}$ M. A. Lieberman and A. J. Lichtenberg, Principles of Plasma Discharges and Materials Processing (Wiley-Interscience, New York, 1994), Chap. 11.

${ }^{21}$ A. Aanesland and Å. Fredriksen, J. Vac. Sci. Technol. A 19, 2446 (2001).

${ }^{22}$ A. Aanesland and A. Fredriksen, Rev. Sci. Instrum. 74, 4336 (2003). 